\title{
Clinical Implication of World Health Organization Classification in Patients with Follicular Thyroid Carcinoma in South Korea: A Multicenter Cohort Study
}

\begin{abstract}
Meihua Jin ${ }^{1, *}$, Eun Sook Kim², , Bo Hyun $\mathrm{Kim}^{3}$, Hee Kyung Kim, Hyon-Seung Yi ${ }^{5}$, Min Ji Jeon ${ }^{1}$, Tae Yong Kim², Ho-Cheol Kang ${ }^{4}$, Won Bae Kim ${ }^{1}$, Young Kee Shong ${ }^{1}$, Mijin Kim³ ${ }^{3}$, Won Gu Kim ${ }^{1}$

${ }^{1}$ Division of Endocrinology and Metabolism, Department of Internal Medicine, Asan Medical Center, University of Ulsan College of Medicine, Seoul; ${ }^{2}$ Department of Internal Medicine, Ulsan University Hospital, University of Ulsan College of Medicine, Ulsan; ${ }^{3}$ Division of Endocrinology and Metabolism, Department of Internal Medicine, Biomedical Research Institute, Pusan National University Hospital, Busan; ${ }^{4}$ Department of Internal Medicine, Chonnam National University Medical School, Gwangju; ${ }^{5}$ Department of Internal Medicine, Chungnam National University Hospital, Daejeon, Korea
\end{abstract}

Background: The study aimed to compare the prognostic value of the 4th edition of World Health Organization classification (WHO-2017) with the previous WHO classification (WHO-2004) for follicular thyroid carcinoma (FTC).

Methods: This multicenter retrospective cohort study included 318 patients with FTC from five tertiary centers who underwent thyroid surgery between 1996 and 2009. We evaluated the prognosis of patients with minimally invasive (MI), encapsulated angioinvasive (EA), and widely invasive (WI) FTC according to WHO-2017. Further, we evaluated the proportion of variation explained (PVE) and Harrell's C-index to compare the predictability of disease-free survival (DFS) and disease-specific survival (DSS).

Results: In total, 227, 58, and 33 patients had MI-, EA-, and WI-FTC, respectively. During a median follow-up of 10.6 years, 46 (14.5\%) patients had disease recurrence and 20 (6.3\%) patients died from FTC. The 10-year DFS rates of patients with MI-, EA-, and WI-FTC were $91.1 \%, 78.2 \%$, and $54.9 \%$, respectively $(P<0.001, \mathrm{PVE}=7.1 \%, \mathrm{C}$-index $=0.649)$. The corresponding 10 -year DSS rates were $95.9 \%, 93.5 \%$, and $73.5 \%$, respectively $(P<0.001, \mathrm{PVE}=2.6 \%, \mathrm{C}$-index $=0.624)$. The $\mathrm{PVE}$ and $\mathrm{C}$-index values were higher using WHO-2017 than using WHO-2004 for the prediction of DFS, but not for DSS. In multivariate analysis, older age $(P=0.02)$, gross extrathyroidal extension (ETE) $(P=0.003)$, and distant metastasis $(P<0.001)$ were independent risk factors for DSS. Conclusion: WHO-2017 improves the predictability of DFS, but not DSS, in patients with FTC. Distant metastasis, gross ETE and older age ( $\geq 55$ years) were independent risk factors for DSS.

Keywords: Thyroid neoplasms; Adenocarcinoma, follicular; World Health Organization; Recurrence; Survival

Received: 22 June 2020, Revised: 5 August 2020, Accepted: 11 August 2020 Corresponding authors: Won Gu Kim

Division of Endocrinology and Metabolism, Department of Internal Medicine, Asan Medical Center, University of Ulsan College of Medicine, 88 Olympic-ro 43-gil, Songpa-gu, Seoul 05505, Korea

Tel: +82-2-3010-5883, Fax: +82-2-3010-6962, E-mail: wongukim@amc.seoul.kr

Mijin Kim

Division of Endocrinology and Metabolism, Department of Internal Medicine, Biomedical Research Institute, Pusan National University Hospital, 179 Gudeokro, Seo-gu, Busan 49241, Korea

Tel: +82-51-240-7738, Fax: +82-51-254-3237, E-mail: mijinkim08@gmail.com

*These authors contributed equally to this work.
Copyright ( $\odot 2020$ Korean Endocrine Society

This is an Open Access article distributed under the terms of the Creative Commons Attribution Non-Commercial License (https://creativecommons.org/ licenses/by-nc/4.0/) which permits unrestricted non-commercial use, distribution, and reproduction in any medium, provided the original work is properly cited. 


\section{INTRODUCTION}

Follicular thyroid carcinoma (FTC) is the second most common subtype of thyroid carcinoma, accounting for approximately $10 \%$ to $15 \%$ cases of differentiated thyroid carcinoma (DTC) in western countries [1]. The incidence is much lower in iodinesufficient area, reported as approximately $3 \%$ to $4 \%$ in a multicenter cohort study in Korea [2,3]. The World Health Organization (WHO) classification of tumors serves an international standard for histopathological diagnosis, and the new 4th edition of the WHO classification (WHO-2017) was published in 2017 [4]. The previous WHO classification (WHO-2004) classified FTC into minimally invasive (MI) and widely invasive (WI) FTC based on the degree of capsular invasion [5]. MI-FTCs have limited capsular and/or vascular invasion, and the invasiveness can only be identified a under microscope. WI-FTCs have widespread infiltration into adjacent thyroid tissue and/or blood vessels [5]. Although this pathological classification of FTC is well accepted, definitions of the extent of capsular invasion varies, and the prognostic significance of vascular invasion remains controversial [6,7]. In WHO-2017, FTC was divided into three prognostic categories, MI-FTC (capsular invasion only), encapsulated angioinvasive FTC (EA-FTC), and WI-FTC $[4,8]$. This new classification highlighted the importance of vascular invasion and the degree of capsular invasion in FTC as important prognostic factors.

FTC shows different biological behaviors and has a poorer prognosis than papillary thyroid carcinoma (PTC) [9]. FTC is common in middle-aged and elderly people and has a higher incidence in iodine-deficient areas [10]. In addition, compared to PTC, FTC tends to have a larger primary tumor size and more frequently metastasizes to distant organs, such as the lungs, bone, and brain [11,12]. Vascular invasion and the number of invaded vessels have prognostic importance in distant metastasis and recurrence $[6,13]$. Older age, larger tumor size, extrathyroidal extension (ETE), cervical lymph node (LN), and distant metastasis have also been identified as independent predictors of recurrence or disease-specific survival (DSS) [14-16]. The new WHO classification is expected to improve the categorization of patients at a high risk of recurrence or death from FTC into more advanced categories.

In this study, we evaluated the prognostic implications of WHO-2017 in patients with FTC using a multi-institutional retrospective cohort. In addition, we elucidated the independent prognostic factors associated with disease-free survival (DFS) and DSS. Further, we compared the prognostic predictability of
DFS and DSS between WHO-2017 and WHO-2004.

\section{METHODS}

\section{Patients}

In this multicenter, retrospective cohort study, 318 consecutive patients with FTC who underwent initial thyroid surgery from January 1996 to December 2009 at five tertiary hospitals in South Korea were included. All patients were pathologically confirmed to have FTC and were aged $>18$ years. Hürthle cell carcinoma, poorly differentiated thyroid carcinoma (PDTC) was excluded in this study. The study protocol was approved by the relevant Institutional Review Boards (Asan Medical Center, 2016-1301; Ulsan University Hospital, 2016-12-031; Pusan National University Hospital, 1701-014-051; Chonnam National University Hwasun Hospital, CNUHH-2017-053, and Chungnam National University Hospital, CNUH 2017-01-018). The need for informed consent was waived owing to the retrospective design of the study.

\section{Definitions}

All patients were classified according to the WHO-2004 and WHO-2017. In WHO-2004, MI-FTCs have limited capsular or vascular invasion, whereas WI-FTCs have widespread infiltration into adjacent thyroid tissue and/or blood vessels [5]. The WHO-2017 further classified patients who were categorized as having MI-FTC according to WHO-2014 into two groups based on the presence of angioinvasion-MI-FTC (capsular invasion only) and EA-FTC [4]. Recurrence was defined as the appearance of pathologically proven malignant tissue and/or the appearance of metastatic lesions. DFS was defined as the time from the date of surgery until recurrence. DSS was defined as the time from the date of surgery until last censoring or death from FTC.

\section{Pathological evaluation}

FTC was diagnosed by the pathologists from each center. The pathologists exam all tumor capsule and check the capsular invasion closely through the deeper section, additionally. Therefore, the possibility of borderline tumor category was not include in this study [17]. PDTC was exclude in this study according to the Turin criteria [18]. We also reviewed the pathology slides of the patients with cervical LN metastasis of FTC to exclude possibility of misdiagnosis such as follicular variant PTC. 


\section{Treatment and follow-up protocol}

During the study period, there were no significant changes in the treatment and follow-up strategy for patients with FTC in South Korea [19-22]. After initial thyroid surgery, patients were regularly followed up every 6 to 12 months and underwent physical examination, thyroid function test, serum thyroglobulin (Tg) measurement, anti-Tg antibody measurement, and neck ultrasonography. Additional diagnostic imaging studies, such as computed tomography, magnetic resonance imaging, or $18 \mathrm{~F}$ fluorodeoxyglucose-positron emission tomography were performed as needed.

\section{Statistical analysis}

Statistical analyses were performed using the R program version 3.5.1 (R Foundation for Statistical Computing, Vienna, Austria; http://www.R-project.org), and survival curves were constructed using Graph Pad Prism version 5.0 (GraphPad Software, SanDiego, CA, USA; http://www.graphpad.com). Continuous variables are presented as mean \pm standard deviation, and categorical variables are presented as numbers (percentages). Survival curves were plotted using the Kaplan-Meier method, and the log-rank test was used to determine survival significance. The relative risks for DFS and DSS were evaluated using the Cox proportional hazards model and presented as hazard ratios (HRs), 95\% confidence intervals (CIs), and $P$ values. The Cox proportional hazard model was used to analyze the prognostic factors associated with DFS and DSS. Backward elimination method was used for the multivariate analysis. The proportion of variation explained (PVE) and Harrell's C-index were calculated to compare the relative validity for predicting DFS and DSS of each pathological classification [23]. The PVE (\%) ranges from 0 to 100 , where a larger number indicates a more accurate predictive model for discriminating the outcome. A model with perfect predictive capacity (sensitivity and specificity of $100 \%$ ) would have a Harrell's C-index of 1.00. A model exhibiting a higher value of the PVE or Harrell's C-index was considered to exhibit a more accurate predictive capacity. All $P$ values were two-sided, and a $P$ value of $<0.05$ was considered statistically significant.

\section{RESULTS}

\section{Baseline characteristics}

The baseline clinical and pathological characteristics of the 318 patients with FTC are listed in Table 1. The mean age at initial diagnosis of FTC was $46.4 \pm 13.7$ years, of the total patients,

\begin{tabular}{lc} 
Table 1. Baseline Characteristics of Patients & with Follicular \\
Thyroid Carcinoma & Value \\
\hline Characteristic & 318 \\
\hline Number & $46.4 \pm 13.7$ \\
Age, yr & $67(21.1)$ \\
Male sex & $3.5 \pm 2.0$ \\
Primary tumor size, cm & \\
Extra-thyroidal extension & $51(16.0)$ \\
Microscopic & $8(2.5)$ \\
Gross & $89(28.0)$ \\
Vascular invasion & $2(0.6)$ \\
Limited vascular invasion $(<4$ foci) & \\
Extensive vascular invasion $(\geq 4$ foci $)$ & $227(71.4)$ \\
WHO classification & $58(18.2)$ \\
Minimally invasive & $33(10.4)$ \\
Encapsulated angioinvasive & $226(71.1)$ \\
Widely invasive & $187(58.8)$ \\
Cervical LN metastasis & $8(2.5)$ \\
N1a & $2(0.6)$ \\
N1b & \\
Distant metastasis & \\
Total thyroidectomy & \\
Radioiodine ablation & \\
\hline Values are expressed as mean \pm standard deviation or number $(\%) .4)$ \\
WHO, World Health Organization; LN, lymph node. \\
\hline
\end{tabular}

$21.1 \%$ were males. The mean size of the primary tumor was $3.5 \pm 2.0 \mathrm{~cm}$, and microscopic ETE and gross ETE were found in $51(16.0 \%)$ and eight (2.5\%) patients, respectively. There was no incomplete resection in all eight patients with gross ETE. Vascular invasion was found in 91 patients $(28.6 \%)$, the majority of which were limited vascular invasion $<4$ foci. At diagnosis, $10(3.1 \%)$ patients had cervical LN metastasis. Of total 318 patients, $30(9.4 \%)$ patients had distant metastasis at initial diagnosis, and newly developed distant metastasis was found in nine patients during the follow-up. Of the 39 patients with distant metastasis, $22(56.4 \%)$ cases were proved by pathology, and 17 $(43.6 \%)$ cases were found on image studies. Approximately $71 \%$ of patients $(n=226)$ underwent total thyroidectomy, and $59 \%$ of patients $(n=187)$ received radioiodine ablation therapy. During a median follow-up of 10.6 years, 46 (14.5\%) patients had recurrent disease and there were 20 (6.3\%) disease-specific deaths. 


\section{DFS according to the WHO classifications, degrees of}

\section{ETE, and vascular invasion}

First, we evaluated the DFS in patients with FTC according to WHO-2004 (Fig. 1A) and WHO-2017 (Fig. 1B). Using WHO2004, the 10-year DFS rates for MI- and WI-FTC were $88.5 \%$ and $54.9 \%$, respectively $(P<0.001)$ (Table 2$)$. Using WHO2017, $227(79.6 \%)$ and $58(20.4 \%)$ patients among those who were classified as having MI-FTC according to WHO-2004 were categorized as having MI- and EA-FTC, respectively. The 10year DFS rates for patients with MI-, EA-, and WI-FTC were 91.1\%, 78.2\%, and 54.9\%, respectively $(P<0.001)$. The PVE value was 5.4\% and 7.1\% using WHO-2004 and WHO-2017, respectively. The Harrell's C-index was also higher using WHO2017 than using WHO-2004 (0.649 vs. 0.391). These findings suggest that WHO-2017 is more useful for predicting DFS in pa-

WHO-2004

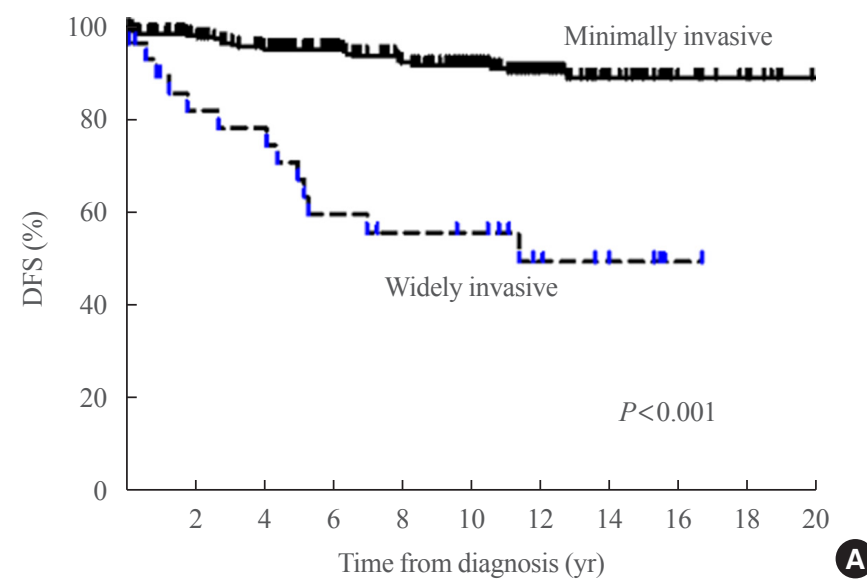

Extrathyroidal extension

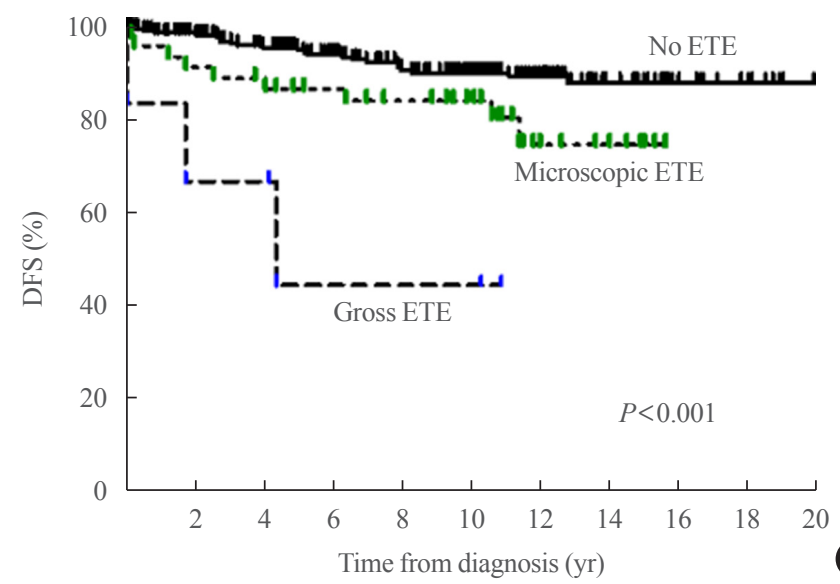

tients with FTC.

We also evaluated the DFS according to the degree of ETE and the presence of vascular invasion (Fig. 1C, D). There was a significant difference in DFS according to the degree of ETE in patients with FTC $(P<0.001)$ (Fig. 1C). The 10-year DFS rates were $87.8 \%, 77.7 \%$, and $38.9 \%$ in patients with no ETE, microscopic ETE, and gross ETE, respectively (PVE $=4.3 \%$, C-in$\mathrm{dex}=0.612$ ) (Table 2). Patients with microscopic and gross ETE had poorer DFS than those with no ETE $(P=0.01$ and $P<0.001$, respectively), and the presence of vascular invasion was significantly associated with poorer DFS $(P<0.001)$ (Fig. 1D). The 10 -year DFS was $91.1 \%$ and $69.9 \%$ in patients with and without vascular invasion, respectively $(\mathrm{PVE}=5.7 \%, \mathrm{C}$-index $=0.351)$. The PVE and the $\mathrm{C}$-index values were the highest using WHO2017 for the prediction of DFS in patients with FTC.
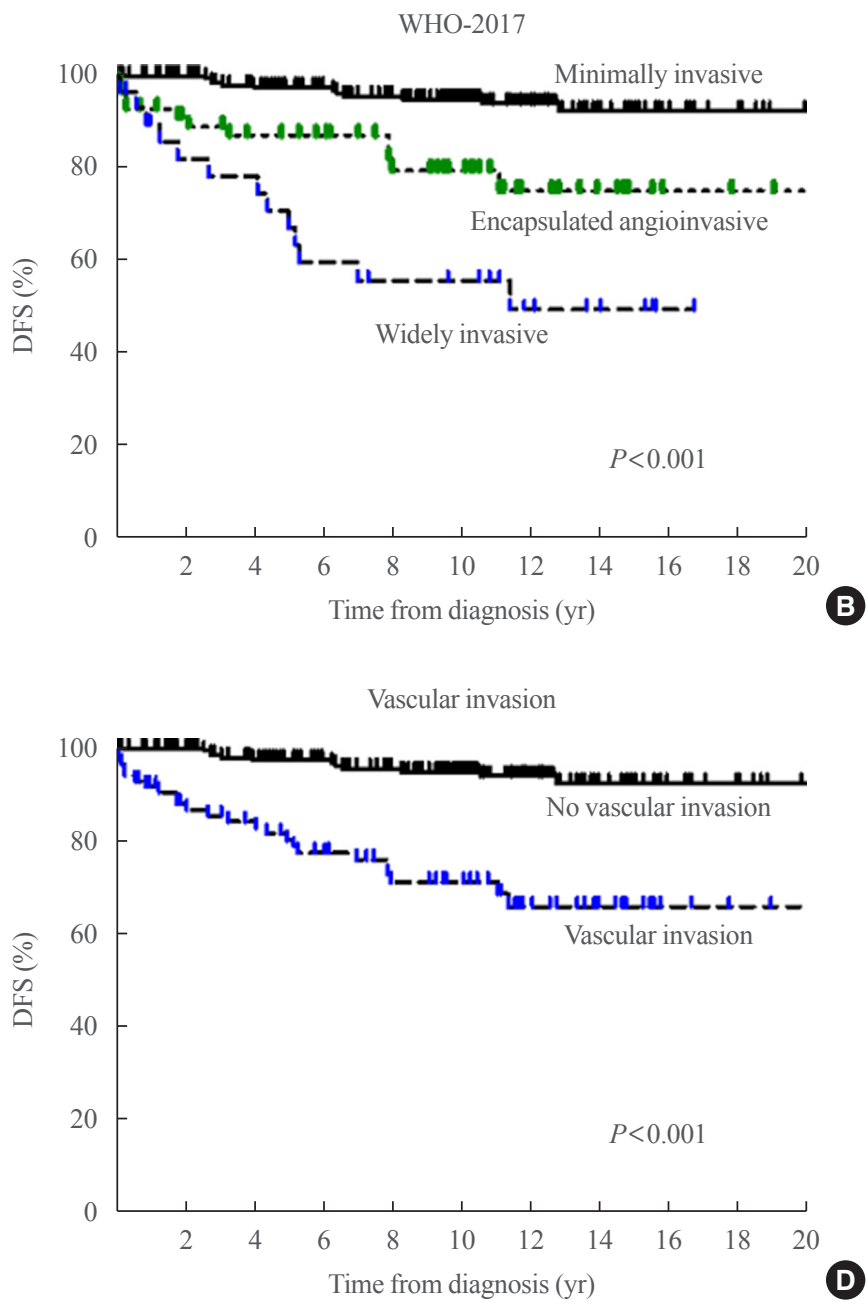

Fig. 1. (A) Disease-free survival curves according to World Health Organization (WHO)-2004. (B) Disease-free survival curves according to WHO-2017. (C) Disease-free survival curves according to the degree of extrathyroidal extension (ETE). (D) Disease-free survival curves according to the presence of vascular invasion. 


\begin{tabular}{|c|c|c|c|c|c|c|c|}
\hline Variable & No. $(\%)$ & $\begin{array}{c}\text { 10-year DFS } \\
\text { rates, } \%\end{array}$ & HR & $95 \% \mathrm{CI}$ & $P$ value & PVE, \% & $\begin{array}{l}\text { Harrell's } \\
\text { C-index }\end{array}$ \\
\hline WHO-2004 & & & & & & 5.4 & 0.391 \\
\hline Minimally invasive & $285(89.6)$ & 88.5 & 1.0 & Reference & & & \\
\hline Widely invasive & $33(10.4)$ & 54.9 & 4.6 & $2.5-8.7$ & $<0.001$ & & \\
\hline WHO-2017 & & & & & & 7.1 & 0.649 \\
\hline Minimally invasive & $227(71.4)$ & 91.1 & 1.0 & Reference & & & \\
\hline Encapsulated angioinvasive & $58(18.2)$ & 78.2 & 2.5 & $1.2-5.1$ & 0.01 & & \\
\hline Widely invasive & $33(10.4)$ & 54.9 & 6.0 & $3.0-11.8$ & $<0.001$ & & \\
\hline ETE & & & & & & 4.3 & 0.612 \\
\hline No ETE & $259(81.4)$ & 87.8 & 1.0 & Reference & & & \\
\hline Micro ETE & $51(16.0)$ & 77.7 & 2.5 & $1.3-4.8$ & 0.007 & & \\
\hline Gross ETE & $8(2.5)$ & 38.9 & 7.6 & $2.6-21.7$ & $<0.001$ & & \\
\hline Vascular invasion & & & & & & 5.7 & 0.351 \\
\hline No vascular invasion & $227(71.4)$ & 91.1 & 1.0 & Reference & & & \\
\hline Vascular invasion & $91(28.6)$ & 69.9 & 3.6 & $2.0-6.5$ & $<0.001$ & & \\
\hline
\end{tabular}

DFS, disease-free survival; HR, hazard ratio; CI, confidence interval; PVE, proportion of variation explained; WHO, World Health Organization; ETE, extrathyroidal extension.

\section{DSS according to the WHO classifications, degrees of ETE, and vascular invasion}

We evaluated the DSS in FTC patients according to the WHO2004 (Fig. 2A) and WHO-2017 (Fig. 2B). Using WHO-2004, the 10 -year DSS rates were $95.4 \%$ and $73.5 \%$ in patients with MI- and WI-FTC, respectively $(P<0.001)$ (Table 3). Using WHO-2017, the 10-year DSS rates were 95.9\%, 93.5\%, and $73.5 \%$ in patients with MI-, EA-, and WI-FTC, respectively $(P<0.001)$. There was no significant difference in DSS between patients with MI-FTC and those with EA-FTC $(P=0.54)$. Only patients with WI-FTC had significantly poorer DSS than those with MI-FTC $(P<0.001)$ using WHO-2017. The PVE value was the same using WHO-2004 and WHO-2017 (2.6\%). The Cindex was 0.366 and 0.624 using WHO-2004 and WHO-2017, respectively (Table 3 ).

The degree of ETE was significantly associated with DSS in patients with FTC $(P<0.001)$ (Fig. 2C). Patients with microscopic ETE and gross ETE had significantly poorer DSS than those with no ETE ( $P=0.01$ and $P<0.001$, respectively). The PVE and C-index was $4.9 \%$ and 0.661 according to the degree of ETE for the prediction of DSS in patients with FTC (Table 3). The presence of vascular invasion was also associated with DSS in patients with FTC $(P=0.03)$ (Fig. 2D). Of the four pathological classifications, the degree of ETE had the highest PVE and
Harrell's C-index for the prediction of DSS.

\section{Clinicopathological features associated with DFS and DSS}

Univariate and multivariate analyses were performed to identify the prognostic factors associated with DFS and DSS in patients with FTC (Tables 4, 5). In multivariate analysis, older age ( $\geq 55$ years; HR, 4.9; 95\% CI, 2.6 to 9.4; $P<0.001$ ), gross ETE (HR, 4.6; $95 \% \mathrm{CI}, 1.5$ to $13.7 ; P=0.01)$, EA-FTC (HR, 2.5; 95\% CI, 1.2 to $5.1 ; P=0.01$ ), WI-FTC (HR, $2.2 ; 95 \% \mathrm{CI}, 1.0$ to $5.0 ; P=$ 0.05 ), and LN metastasis (HR, 2.9; 95\% CI, 1.0 to $7.9 ; P=0.04$ ) were significantly associated with DFS (Table 4). Larger tumor size $(>4 \mathrm{~cm})$ was associated with univariate analysis, but it was not remained as independent risk factor in multivariate analysis. Older age ( $\geq 55$ years; HR, $1.3 ; 95 \% \mathrm{CI}, 1.1$ to $11.8 ; P=0.03$ ), gross ETE (HR, 9.3; 95\% CI, 2.2 to 39.9; $P=0.002)$ and distant metastasis (HR, 30.0; 95\% CI, 9.1 to 98.3 ; $P<0.001$ ) were independent prognostic factors for DSS in patients with FTC (Table 5). WI-FTC and LN metastasis were associated with DSS only in univariate analysis, but not in the multivariate analysis.

\section{DISCUSSION}

In this retrospective multicenter cohort study, we evaluated the prognostic value of WHO-2017 and independent prognostic 
WHO-2004

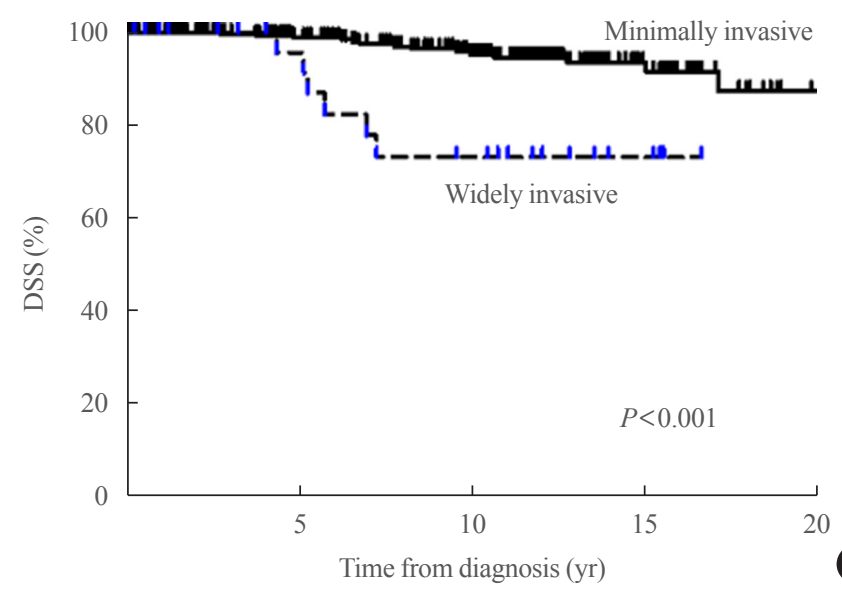

Extrathyroidal extension

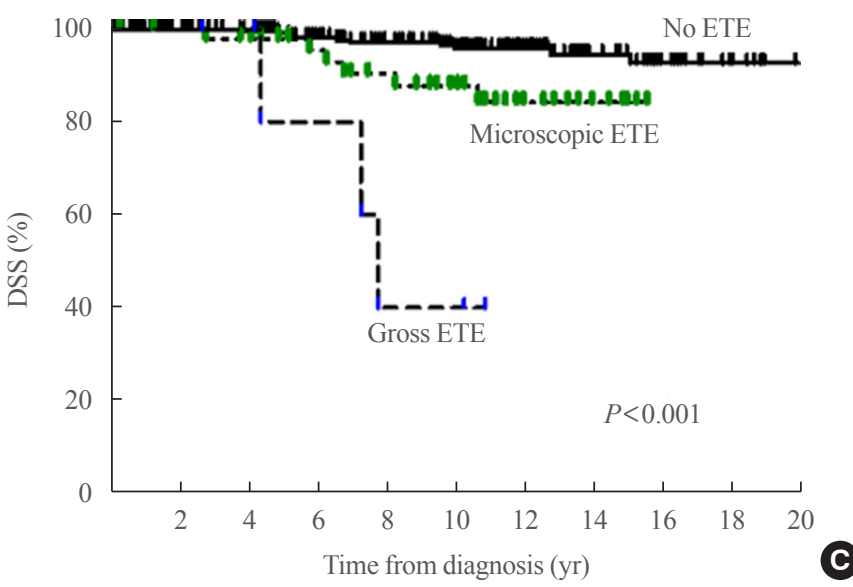

WHO-2017
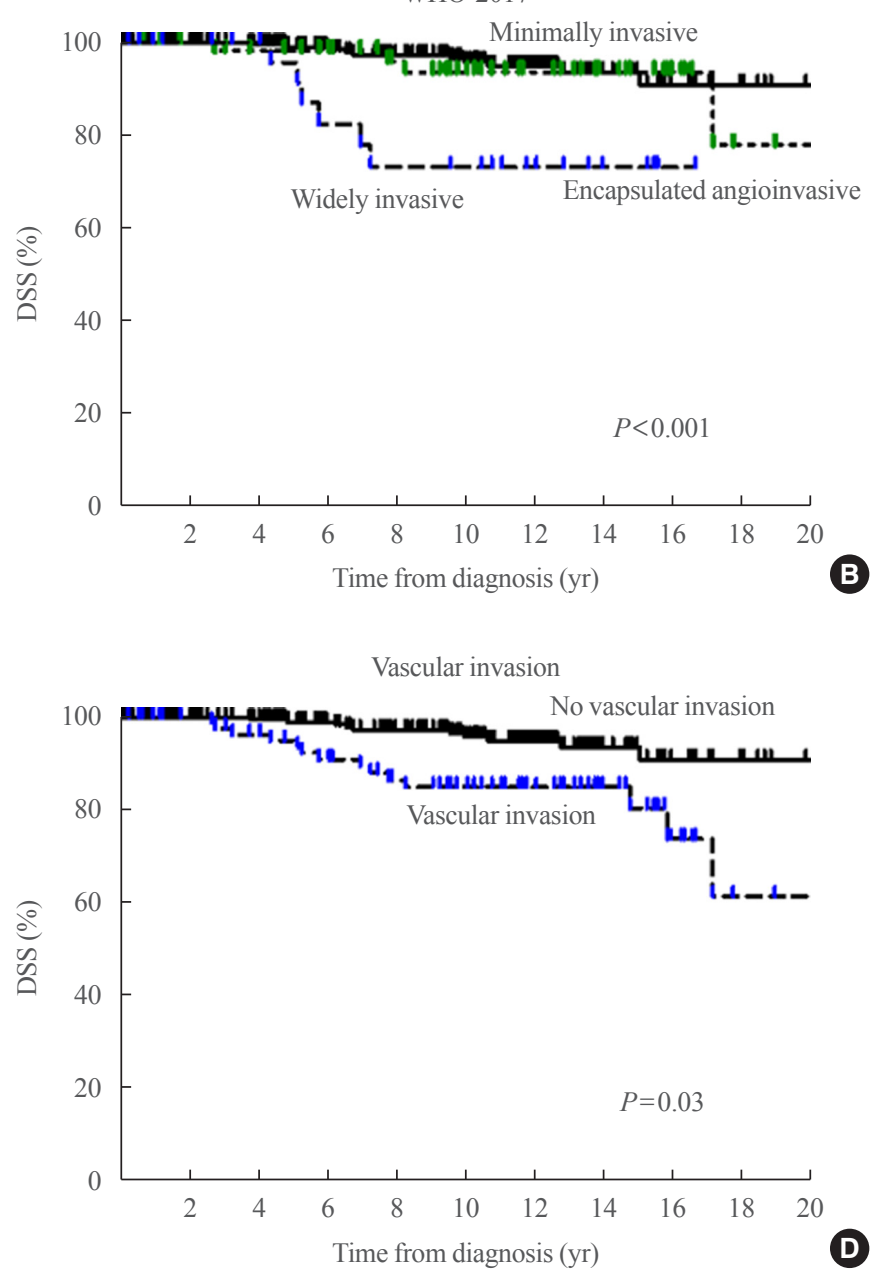

Fig. 2. (A) Disease-specific survival curves according to World Health Organization (WHO)-2004. (B) Disease-specific survival curves according to WHO-2017. (C) Disease-specific survival curves according to the degree of extrathyroidal extension (ETE). (D) Disease-specific curves according to the presence of vascular invasion.

factors associated with DFS and DSS in 318 patients with FTC. After adapting WHO-2017, 79.6\% and 20.4\% patients who were classified as having MI-FTC based on WHO-2004 were categorized as having MI-FTC and EA-FTC, respectively. Using WHO-2017, patients with EA- and WI-FTC had significantly poorer DFS than those with MI-FTC, and there was significant difference in the DFS curves among groups. Comparing WHO-2004 and WHO-2017, the PVE and C-index were increased from $5.4 \%$ to $7.1 \%$ and 0.391 to 0.649 , respectively. However, there was no significant difference in DSS between patients with MI- and EA-FTC. Only patients with WI-FTC had significantly poorer DSS than those with MI-FTC. The PVE value was $2.6 \%$ using both WHO-2004 and WHO-2017. Independent risk factors associated with shorter DFS were older age, gross ETE, EA- and WI-FTC, and presence of LN metastasis.
In terms of DSS, older age, gross ETE, and distant metastasis were significantly associated with shorter DSS. To the best of our knowledge, this is the first large multicenter cohort study evaluating the prognostic value of WHO-2017 in an iodine-sufficient area in South Korea.

In WHO-2017, the extent of invasiveness and the presence of vascular invasion are important factors in classifying patients with FTC [4]. In a previous retrospective study, including 132 patients with FTC, patients with MI-FTC (capsular invasion only) had a slightly better 5-year survival rate (98\%) than patients who had angioinvasion with or without capsular invasion (80\%) and WI-FTC (38\%) [24]. A prospective study by O'Neill et al. [7] evaluated 124 patients with FTC over a median followup of 40 months and reported a significant difference in DFS between the MI-FTC, EA-FTC, and WI-FTC groups. Based on 


\begin{tabular}{|c|c|c|c|c|c|c|c|}
\hline Variable & No. $(\%)$ & $\begin{array}{c}10 \text {-year DSS } \\
\text { rates, \% }\end{array}$ & HR & $95 \% \mathrm{CI}$ & $P$ value & PVE, \% & $\begin{array}{l}\text { Harrell's } \\
\text { C-index }\end{array}$ \\
\hline WHO-2004 & & & & & & 2.6 & 0.366 \\
\hline Minimally invasive & $285(89.6)$ & 95.4 & 1.0 & Reference & & & \\
\hline Widely invasive & $33(10.4)$ & 73.5 & 5.1 & $2.0-13.6$ & $<0.001$ & & \\
\hline WHO-2017 & & & & & & 2.6 & 0.624 \\
\hline Minimally invasive & $227(71.4)$ & 95.9 & 1.0 & Reference & & & \\
\hline Encapsulated angioinvasive & $58(18.2)$ & 93.5 & 1.4 & 0.44 .6 & 0.54 & & \\
\hline Widely invasive & $33(10.4)$ & 73.5 & 5.6 & $2.1-15.6$ & $<0.001$ & & \\
\hline ETE & & & & & & 4.9 & 0.661 \\
\hline No ETE & $259(81.4)$ & 96.0 & 1.0 & Reference & & & \\
\hline Micro ETE & $51(16.0)$ & 88.0 & 3.9 & $1.5-10.4$ & 0.01 & & \\
\hline Gross ETE & $8(2.5)$ & 40.0 & 19.9 & $5.3-75.0$ & $<0.001$ & & \\
\hline Vascular invasion & & & & & & 1.4 & 0.376 \\
\hline No vascular invasion & $227(71.4)$ & 95.9 & 1.0 & Reference & & & \\
\hline Vascular invasion & 91 (28.6) & 87.2 & 2.6 & $1.1-6.2$ & 0.03 & & \\
\hline
\end{tabular}

DSS, disease-specific survival; HR, hazard ratio; CI, confidence interval; PVE, proportion of variation explained; WHO, World Health Organization; ETE, extrathyroidal extension.

Table 4. Clinicopathological Features Associated with Disease-Free Survival in Patients with Follicular Thyroid Carcinoma

\begin{tabular}{|c|c|c|c|c|}
\hline \multirow{2}{*}{ Variable } & \multicolumn{2}{|c|}{ Univariate } & \multicolumn{2}{|c|}{ Multivariate } \\
\hline & HR $(95 \% \mathrm{CI})$ & $P$ value & $\mathrm{HR}(95 \% \mathrm{CI})$ & $P$ value \\
\hline Older age ( $\geq 55 \mathrm{yr})$ & $6.3(3.4-11.5)$ & $<0.001$ & $4.9(2.6-9.4)$ & $<0.001$ \\
\hline Male sex & $1.2(0.6-2.3)$ & 0.67 & & \\
\hline Larger size $(>4 \mathrm{~cm})$ & $2.1(1.2-3.8)$ & 0.012 & & \\
\hline \multicolumn{5}{|l|}{ ETE (ref. no ETE) } \\
\hline Micro ETE & $2.5(1.3-4.8)$ & 0.007 & $1.4(0.7-2.8)$ & 0.34 \\
\hline Gross ETE & $7.6(2.6-21.7)$ & $<0.001$ & $4.6(1.5-13.7)$ & 0.01 \\
\hline \multicolumn{5}{|c|}{ WHO classification (ref. minimally invasive) } \\
\hline Encapsulated angioinvasive & $2.5(1.2-5.1)$ & 0.012 & $2.5(1.2-5.1)$ & 0.01 \\
\hline Widely invasive & $6.0(3.0-11.8)$ & $<0.001$ & $2.2(1.0-5.0)$ & 0.05 \\
\hline LN metastasis & $6.8(2.9-16.3)$ & $<0.001$ & $2.9(1.0-7.9)$ & 0.04 \\
\hline
\end{tabular}

HR, hazard ratio; CI, confidence interval; ETE, extrathyroidal extension; WHO, World Health Organization; LN, lymph node.

these findings, these pathological classifications were adopted in WHO-2017 to provide more realistic information for the prognosis of patients with FTC [8]. In our study, the 10-year DFS rate of patients with MI-FTC was significantly lower than that of patients with EA-FTC. Considering the higher PVE and C-index values for DFS, WHO-2017 improves the predictability for recurrence compared to WHO-2004, although WHO2017 fails to show better predictability for DSS. Further studies are needed to evaluate the prognostic value of WHO-2017 in patients with FTC.

In our study, multivariate analysis showed that gross ETE was an independent prognostic factor for both DFS and DSS in patients with FTC. In addition, the recurrence was increased 7-fold (HR, 7.6; 95\% CI, 2.6 to 21.7) and disease-specific death was increased 19-fold (HR, 19.9; 95\% CI, 5.3 to 75.0) in patients with gross ETE compared to patients with no ETE. To 
Table 5. Clinicopathological Features Associated with Disease-Specific Survival in Patients with Follicular Thyroid Carcinoma

\begin{tabular}{|c|c|c|c|c|}
\hline \multirow{2}{*}{ Variable } & \multicolumn{2}{|c|}{ Univariate } & \multicolumn{2}{|c|}{ Multivariate } \\
\hline & $\operatorname{HR}(95 \% \mathrm{CI})$ & $P$ value & $\operatorname{HR}(95 \% \mathrm{CI})$ & $P$ value \\
\hline Older age $(\geq 55 \mathrm{yr})$ & $9.6(3.5-26.4)$ & $<0.001$ & $1.3(1.1-11.8)$ & 0.03 \\
\hline Male sex & $1.0(0.3-3.1)$ & 0.95 & & \\
\hline Larger size $(>4 \mathrm{~cm})$ & $2.2(0.9-5.3)$ & 0.08 & & \\
\hline \multicolumn{5}{|l|}{ ETE (ref. no ETE) } \\
\hline Micro ETE & $3.9(1.5-10.4)$ & 0.006 & $1.0(0.4-2.9)$ & 0.94 \\
\hline Gross ETE & $19.9(5.3-75.0)$ & $<0.001$ & $9.3(2.2-39.9)$ & 0.002 \\
\hline \multicolumn{5}{|c|}{ WHO classification (ref. minimally invasive) } \\
\hline Encapsulated angioinvasive & $1.4(0.5-4.6)$ & 0.55 & & \\
\hline Widely invasive & $5.6(2.0-15.6)$ & $<0.001$ & & \\
\hline LN metastasis & $7.4(1.7-32.7)$ & 0.01 & & \\
\hline Distant metastasis & $56.5(18.8-169.9)$ & $<0.001$ & $30.0(9.1-98.3)$ & $<0.001$ \\
\hline
\end{tabular}

HR, hazard ratio; CI, confidence interval; ETE, extrathyroidal extension; WHO, World Health Organization; LN, lymph node.

date, limited studies have focused on the degree of gross ETE in patients with FTC. Some previous studies have evaluated ETE as the prognostic factor in patients with DTC, including FTC, but the majority of the cases were PTC. Furthermore, other studies have evaluated ETE in patients with FTC, but the ETE generally included both microscopic and gross ETE $[14,15,24-$ 27]. In a previous study, including 558 patients with DTC, ETE was observed in $4.9 \%$ of 81 patients with FTC and was considered as an independent risk factor in predicting DFS in patients with FTC [27]. In a retrospective cohort study including 438 patients with FTC in South Korea, ETE was an independent risk factor for distant metastasis, but not for recurrence [14]. However, these studies included both microscopic and gross ETE when evaluating the prognosis [14,27]. In our study, gross ETE was observed in $2.5 \%$ patients with FTC and was considered an independent prognostic factor for predicting both recurrence and disease-specific death. Conversely, microscopic ETE was observed in $16.0 \%$ patients and was associated with DFS and DSS in univariate analysis, but not in multivariate analysis.

In our study, distant metastasis at the time of diagnosis was an important prognostic factor in patients with FTC, which is in accordance with the result of previous studies $[15,28]$. It is generally accepted that distant metastasis rates are higher in FTC than in PTC [11,12]. In our study, of the 318 patients, 30 (9.4\%) patients had distant metastasis at the time of diagnosis. Although distant metastasis in FTC are not uniformly fatal, disease-specific death in FTC is rare without distant metastasis [15]. Many investigators assume that the risk of distant metasta- sis increases with pathological progression from capsule invasion alone to minimal vascular invasion ( $<4$ blood vessels) to WI with extensive vascular invasion ( $>4$ blood vessels) or gross ETE [15], which is consistent with the findings of our study. Of the 30 patients with distant metastasis at diagnosis, 10 patients had MI-FTC (10 of 227 patients, 4.4\%), six patients had EAFTC (six of 58 patients, 10.3\%), and 14 patients had WI-FTC (14 of 33 patients, $42.4 \%$ ). The proportion of patients with distant metastasis significantly increased according to WHO-2017 ( $\mathrm{P}$ for trend $<0.001$ ). This direct association between WHO2017 and distant metastasis may explain the reason why WHO2017 was associated with DSS in univariate analysis, but not in multivariate analysis.

This study has several limitations. First, this study had a retrospective design and involved a relatively small number of patients with FTC since the prevalence of FTC is relatively low in iodine-sufficient areas. Second, there is a possibility of selection bias because we included patients from five tertiary referral centers, and there may be some differences in follow-up and therapeutic strategies between the five centers. Third, centralized pathological examination was not determined in this multicenter study and we also did not excluded PDTC by the definition based on mitosis and necrosis all the centers [29]. However, this is the first multicenter cohort study to evaluate the prognostic value of WHO-2017 in an iodine-sufficient area in South Korea.

In conclusion, WHO-2017 improves the predictability of DFS in patients with FTC compared to WHO-2004. Older age, distant metastasis and gross ETE are independent prognostic fac- 
tors for predicting DSS in patients with FTC. These findings suggest that WHO-2017, the presence of ETE, distant metastasis and patients' age should be considered while selecting the follow-up and treatment strategies in patients with FTC.

\section{CONFLICTS OF INTEREST}

No potential conflict of interest relevant to this article was reported.

\section{ACKNOWLEDGMENTS}

This study was supported by a Korean Endocrine Society EnM Research Award 2019.

\section{AUTHOR CONTRIBUTIONS}

Conception or design: M.J., E.S.K., B.H.K., H.K.K., H.S.Y., M.J.J., T.Y.K., H.C.K., W.B.K., Y.K.S., M.K., W.G.K. Acquisition, analysis, or interpretation of data: M.J., E.S.K., B.H.K., H.K.K., H.S.Y., M.J.J., T.Y.K., H.C.K., W.B.K., Y.K.S., M.K., W.G.K. Drafting the work or revising: M.J., E.S.K., M.K., W.G.K. Final approval of the manuscript: M.J., E.S.K., M.K., W.G.K.

\section{ORCID}

Meihua Jin https://orcid.org/0000-0003-0196-631X

Eun Sook Kim https://orcid.org/0000-0002-9311-4642

Mijin Kim https://orcid.org/0000-0002-1538-8859

Won Gu Kim https://orcid.org/0000-0002-8404-7759

\section{REFERENCES}

1. Dralle H, Machens A, Basa J, Fatourechi V, Franceschi S, Hay ID, et al. Follicular cell-derived thyroid cancer. Nat Rev Dis Primers 2015;1:15077.

2. Jeon MJ, Kim WG, Kim TH, Kim HK, Kim BH, Yi HS, et al. Disease-specific mortality of differentiated thyroid cancer patients in Korea: a multicenter cohort study. Endocrinol Metab (Seoul) 2017;32:434-41.

3. Jeon MJ, Kim HK, Kim EH, Kim ES, Yi HS, Kim TY, et al. Decreasing disease-specific mortality of differentiated thyroid cancer in Korea: a multicenter cohort study. Thyroid 2018; 28:1121-7.

4. LIoyd RV, Osamura RY, Kloppel G, Rosai J. WHO classifi- cation of tumours of endocrine organs. Lyon: International Agency for Research on Cancer; 2017.

5. DeLellis RA. Pathology and genetics of tumours of endocrine organs. Lyon: International Agency for Research on Cancer; 2004.

6. Collini P, Sampietro G, Pilotti S. Extensive vascular invasion is a marker of risk of relapse in encapsulated nonHurthle cell follicular carcinoma of the thyroid gland: a clinicopathological study of 18 consecutive cases from a single institution with a 11-year median follow-up. Histopathology 2004;44:35-9.

7. O’Neill CJ, Vaughan L, Learoyd DL, Sidhu SB, Delbridge LW, Sywak MS. Management of follicular thyroid carcinoma should be individualised based on degree of capsular and vascular invasion. Eur J Surg Oncol 2011;37:181-5.

8. Kakudo K, Bychkov A, Bai Y, Li Y, Liu Z, Jung CK. The new 4th edition World Health Organization classification for thyroid tumors, Asian perspectives. Pathol Int 2018;68:64164.

9. Aschebrook-Kilfoy B, Grogan RH, Ward MH, Kaplan E, Devesa SS. Follicular thyroid cancer incidence patterns in the United States, 1980-2009. Thyroid 2013;23:1015-21.

10. Grebe SK, Hay ID. Follicular thyroid cancer. Endocrinol Metab Clin North Am 1995;24:761-801.

11. DeGroot LJ, Kaplan EL, Shukla MS, Salti G, Straus FH. Morbidity and mortality in follicular thyroid cancer. J Clin Endocrinol Metab 1995;80:2946-53.

12. Passler C, Scheuba C, Prager G, Kaczirek K, Kaserer K, Zettinig G, et al. Prognostic factors of papillary and follicular thyroid cancer: differences in an iodine-replete endemic goiter region. Endocr Relat Cancer 2004;11:131-9.

13. Ghossein R. Problems and controversies in the histopathology of thyroid carcinomas of follicular cell origin. Arch Pathol Lab Med 2009;133:683-91.

14. Kim WG, Kim TY, Kim TH, Jang HW, Jo YS, Park YJ, et al. Follicular and Hurthle cell carcinoma of the thyroid in iodine-sufficient area: retrospective analysis of Korean multicenter data. Korean J Intern Med 2014;29:325-33.

15. Daniels GH. Follicular thyroid carcinoma: a perspective. Thyroid 2018;28:1229-42.

16. Grani G, Lamartina L, Durante C, Filetti S, Cooper DS. Follicular thyroid cancer and Hurthle cell carcinoma: challenges in diagnosis, treatment, and clinical management. Lancet Diabetes Endocrinol 2018;6:500-14.

17. Zhu Y, Li Y, Jung CK, Song DE, Hang JF, Liu Z, et al. Histopathologic assessment of capsular invasion in follicular 
thyroid neoplasms-an observer variation study. Endocr Pathol 2020;31:132-40.

18. Volante M, Collini P, Nikiforov YE, Sakamoto A, Kakudo K, Katoh R, et al. Poorly differentiated thyroid carcinoma: the Turin proposal for the use of uniform diagnostic criteria and an algorithmic diagnostic approach. Am J Surg Pathol 2007; 31:1256-64.

19. Singer PA, Cooper DS, Daniels GH, Ladenson PW, Greenspan FS, Levy EG, et al. Treatment guidelines for patients with thyroid nodules and well-differentiated thyroid cancer. American Thyroid Association. Arch Intern Med 1996;156: 2165-72.

20. Yi KH. The revised 2016 Korean Thyroid Association guidelines for thyroid nodules and cancers: differences from the 2015 American Thyroid Association guidelines. Endocrinol Metab (Seoul) 2016;31:373-8.

21. Yi KH, Park YJ, Koong SS, Kim JH, Na DG, Ryu JS, et al. Revised Korean Thyroid Association management guidelines for patients with thyroid nodules and thyroid cancer. Korean J Otorhinolaryngol-Head Neck Surg 2011;54:8-36.

22. Kim WB, Kim TY, Kwon HS, Moon WJ, Lee JB, Choi YS, et al. Management guidelines for patients with thyroid nodules and thyroid cancer. J Korean Endocr Soc 2007;22:15787.
23. Lang BH, Lo CY, Chan WF, Lam KY, Wan KY. Staging systems for papillary thyroid carcinoma: a review and comparison. Ann Surg 2007;245:366-78.

24. D’Avanzo A, Treseler P, Ituarte PH, Wong M, Streja L, Greenspan FS, et al. Follicular thyroid carcinoma: histology and prognosis. Cancer 2004;100:1123-9.

25. Ito Y, Hirokawa M, Higashiyama T, Takamura Y, Miya A, Kobayashi K, et al. Prognosis and prognostic factors of follicular carcinoma in Japan: importance of postoperative pathological examination. World J Surg 2007;31:1417-24.

26. Lo CY, Chan WF, Lam KY, Wan KY. Follicular thyroid carcinoma: the role of histology and staging systems in predicting survival. Ann Surg 2005;242:708-15.

27. Aboelnaga EM, Ahmed RA. Difference between papillary and follicular thyroid carcinoma outcomes: an experience from Egyptian institution. Cancer Biol Med 2015;12:53-9.

28. Sugino K, Ito K, Nagahama M, Kitagawa W, Shibuya H, Ohkuwa K, et al. Prognosis and prognostic factors for distant metastases and tumor mortality in follicular thyroid carcinoma. Thyroid 2011;21:751-7.

29. Hiltzik D, Carlson DL, Tuttle RM, Chuai S, Ishill N, Shaha A, et al. Poorly differentiated thyroid carcinomas defined on the basis of mitosis and necrosis: a clinicopathologic study of 58 patients. Cancer 2006;106:1286-95. 